TRANSACTIONS OF THE

AMERICAN MATHEMATICAL SOCIETY

Volume 351, Number 12, Pages 4693-4711

S 0002-9947(99)02511-8

Article electronically published on August 25, 1999

\title{
SYMMETRY OF PROPERLY EMBEDDED SPECIAL WEINGARTEN SURFACES IN $\mathbf{H}^{3}$
}

\author{
RICARDO SA EARP AND ERIC TOUBIANA
}

\begin{abstract}
In this paper we prove some existence and uniqueness results about special Weingarten surfaces in hyperbolic space.
\end{abstract}

\section{INTRODUCTION}

In this paper we consider $C^{2}$ surfaces $M$ in $\mathbf{H}^{3}$ oriented by a global unit normal field $N$ whose mean curvature $H$ and extrinsic Gaussian curvature $K_{e}$ satisfy a Weingarten relation of the form

$$
H=f\left(H^{2}-K_{e}\right) .
$$

We shall require that $f$ is a $C^{1}$ function defined on $[0,+\infty[$, satisfying

$$
\forall t \in\left[0,+\infty\left[, 4 t f^{\prime 2}(t)<1 .\right.\right.
$$

We will say that $f$ is elliptic if $f$ satisfies inequality (2). If $M$ satisfies relation (1) for $f$ elliptic, we will call $M$ either a special Weingarten surface or an $f$-surface. In euclidean space they have been studied by Hopf [10], Hartman and Wintner [9], Chern [5]. More recently, there has been great progress, as much in the euclidean case as in the hyperbolic, Bryant [4], Braga Brito [3], Rosenberg [15]. In section 2 we will briefly describe this and we will develop the fundamental basis of the theory. Both authors have constructed a family of embedded (and also a family of non-embedded) complete rotational Weingarten surfaces in $\mathbf{R}^{3}$. They have also proved uniqueness of such surfaces (see [16], [17] and [18]).

An interesting question in this subject is whether or not an $f$-surface inherits the symmetries of its boundary: When $f=H$ (constant) and $|H| \leq 1$, Nelli and Rosenberg [14] solved the boundary value problem for compact embedded surfaces and spherical boundary. The sharp related theorem for $M$ immersed was proved by Barbosa and the first author in [2]. Here, we shall generalize Rosenberg and Nelli's result to $f$-surfaces when $f^{2} \leq 1$.

In [1], it is stated that when the boundary consists of two circles invariant under a 1-parameter group of rotations of $\mathbf{H}^{3}$, then stability implies the surface is also invariant under rotations. Barbosa and the first author have asked whether the assumption of stability could be replaced by embeddedness [1]. In this direction, we are able to obtain the following result: Assume $\partial M=C_{1} \cup C_{2}$, where $C_{1}$ and $C_{2}$ are circles of the same radius. Suppose that $\partial M$ is invariant under a rotation. Then there is a positive real number $d_{0}$ such that if $|H|=1$ and $\operatorname{dist}\left(C_{1}, C_{2}\right) \geq 2 d_{0}$,

Received by the editors March 18, 1996.

1991 Mathematics Subject Classification. Primary 53A05, 53C42.

Both authors were partially supported by CNPq and FAPERJ, Brazil. 
then $M$ is a piece of a Catenoid Cousin. In fact, we prove an analogous result for a wider class of $f$-surfaces (see Theorem 1, section 3).

We mention that symmetry results proved by several authors concerning $\mathrm{H}$ surfaces whose asymptotic boundary consists of a point, a circle or the union of two circles ([6], [7], [13]) are valid for $f$-surfaces, since the maximum principle still holds (see section 2). On the other hand, others characterizations of $f$-surfaces with boundary a circle, proved in [3] when the ambient space is euclidean space, are also stated in section 2 .

Finally, we will give existence and uniqueness results for rotational $f$-surfaces in section 4, under further hypotheses on $f$. Moreover, we will prove that the geometric behaviour of such surfaces is the same as that of the related well-known rotational $H$-surfaces in $\mathbf{H}^{3}$ (see Theorems 2 and 3).

\section{Some BASIC PROPERTIES: THE STRUCTURE OF PRINCIPAL Lines AND THE MAXIMUM PRINCIPLE. IMMEDIATE CONSEQUENCES AND GENERALIZATIONS}

In this section we shall establish some basic properties of special Weingarten surfaces. We will state some results of Bryant's theory [4] on the structure of the principal line distribution and we will write down the maximum principle. We will point out that one can apply Alexandrov techniques to obtain immediate consequences generalizing well-known results for the case $H=$ const.

2.1. Principal lines distribution (R. Bryant's theory). Let $M$ be an immersed special Weingarten surface satisfying (1) with respect to a global unit normal field $N$.

In a pioneering paper, R. Bryant had an amazing idea of constructing a new metric $d s^{2}$ by means of the function $f$ given in the Weingarten relation (1) to obtain constant mean curvature type results, as follows:

Proposition $1([4])$. Let $z$ be an isothermal coordinate of the Bryant metric $d s^{2}$ in $U$. If $d s^{2}=\lambda^{2}|d z|^{2}$ then the function $\phi(z)=\lambda^{2}(z)\left(\frac{l-n}{2}-i m\right)$ is holomorphic, where $l, m$, and $n$ are the coefficients of the second fundamental form with respect to a local oriented orthonormal frame field. Furthermore, the holomorphic quadratic form $\Phi(z)=\phi(z)(d z)^{2}$ is defined globally on $M$.

Proposition $2([4])$. Either $M$ is totally umbilic, or else the umbilic locus consists entirely of isolated points of strictly negative index.

We remark that when $f$ is analytic Proposition 2 follows from the proof of theorem 3.2 on p. 142 of Hopf's famous book [10]. Notice that $z_{0}$ is an umbilic point of $M$ if and only if $\phi\left(z_{0}\right)=0$. Thus, in a neighborhood of $z_{0}=0$ the holomorphic quadratic form is $z^{n}(d z)^{2}$; hence the index at $z_{0}$ is $\frac{-n}{2}$.

We emphasize that R. Bryant proved (in view of Propositions 1,2) the following generalization of Hopf's theorem: A closed genus zero special Weingarten surface satisfying (1) is a round sphere.

Remark 1. We can derive from Bryant's construction and straightforward computations that the principal line distribution is given by $\operatorname{Im} \Phi=0$. 
2.2 Maximum principle. In this section we will establish interior and boundary maximum principles for special Weingarten surfaces in hyperbolic space. We shall apply these maximum principles in subsequent sections to derive several uniqueness and symmetry results.

Lemma 1 (Interior maximum principle). Suppose $M_{1}, M_{2}$ are $C^{2}$ surfaces in $\mathbf{H}^{3}$ which are given locally as graphs of $C^{2}$ functions $u, v$. Suppose the tangent planes of both $M_{1}$ and $M_{2}$ agree at a point $p$.

Let $H\left(N_{1}\right)$ and $H\left(N_{2}\right)$ be the mean curvature functions of $u$ and $v$ with respect to unit normals $N_{1}$ and $N_{2}$ that agree at $p$. Let $K_{i}$ be the extrinsic Gaussian curvature of $M_{i}, i=1,2$. Suppose the $M_{i}$ satisfy

$$
H\left(N_{i}\right)=f\left(H_{i}{ }^{2}-K_{i}\right), \quad i=1,2,
$$

for $f$ elliptic.

Then, if $u \leq v$ near $p$, also $M_{1}=M_{2}$ near $p$.

Lemma 2 (Boundary maximum principle). Consider $M_{1}, M_{2}$ as in the statement of the interior maximum principle with $C^{2}$ boundaries $B_{1}, B_{2}$ given by restritions of $u$ and $v$. Suppose $T_{p} M_{1}=T_{p} M_{2}$ and $T_{p} B_{1}=T_{p} B_{2}$ with $p$ in the interior of both $B_{1}$ and $B_{2}$. Suppose $M_{1}, M_{2}$ satisfy (1) and (2) with respect to the same normal $N$ at $p$.

Then $u \leq v$ near $p$ implies $M_{1}=M_{2}$ near $p$.

The analogous statement in euclidean space is proved in [3], so we omit the proof here.

2.3. Immediate consequences. In this section we will derive some applications extending known results of the constant mean curvature theory.

Proposition: Alexandrov theorem for special Weingarten surfaces in hyperbolic space. If $M$ is a closed embedded special Weingarten surface in $\mathbf{H}^{3}$ satisfying (1) for $f$ elliptic, then $M$ is a sphere.

The proof makes use of the Alexandrov reflection principle, with totally geodesic hyperbolic planes in place of ordinary planes in the Euclidean case. For Alexandrov techniques the reader is referred to [3], [13] and [15].

The Alexandrov reflection principle is a beautiful and fundamental tool for several nice theorems in the theory of constant mean curvature surfaces. It provides several analogous results for special Weingarten surfaces. At this point, we will make use of the unit ball model for the hyperbolic space $\mathbf{H}^{3}$. Thus, $\overline{\mathbf{H}^{3}}$ will denote the closed unit ball and $S^{2}(\infty)$ will denote the unit sphere. We will recall some basic definitions given in [7]. Let $M$ be an embedded surface in $\mathbf{H}^{3}$. We denote by $\partial_{\infty} M$ the intersection of the closure of $M$ with $S^{2}(\infty)$, and we will call $\partial_{\infty} M$ the asymptotic boundary of $M$. We will say $M$ is $C^{2}$-regular at infinity if $\bar{M} \subset \overline{\mathbf{H}^{3}}$ is a $C^{2}$ surface with boundary in $\overline{\mathbf{H}^{3}}$ and $\partial_{\infty} M$ is a $C^{2}$ curve of $S^{2}(\infty)$.

Now we set up the following symmetry statements. Let $M$ be a connected complete properly embedded surface in $\mathbf{H}^{3}$. Suppose $M$ is a special Weingarten surface satisfying (1) for $f$ elliptic. Assume $M$ is $C^{2}$-regular at infinity. Then:

(1) If the asymptotic boundary is one point, then $M$ is a horosphere.

(2) If the asymptotic boundary is one circle, then $M$ is a geodesic plane or a equidistant surface. 
(3) If the asymptotic boundary is the union of two disjoint circles, then $M$ is a surface of revolution.

The proof is an application of the Alexandrov principle as in the constant mean curvature case, so we omit the proof here. Let us give the references for the above statements in the case $H=$ const. If $H=0$ and $M$ is immersed, statement 3 was proved by Levitt and Rosenberg [13]. Statements 1 and 2 was derived by Do Carmo and Lawson [6], while statement 3 for $H=$ const $\neq 0$ was deduced by Do Carmo, Gomes and Thorbergsson [7].

Another amazing symmetry result for $H=$ const is the Hsiang theorem, which implies that if $M$ is cylindrically bounded, complete and embedded then $M$ has rotational symmetry [11]. We now assert the following: Let $M$ be a connected properly embedded complete special Weingarten surface in $\mathbf{H}^{3}$ satisfying (1) for $f$ elliptic. If $M$ is cylindrically bounded then $M$ is a surface of revolution.

The proof is similar to the constant mean curvature case.

\section{Symmetry ARISING FROM the BOUNDARY}

In this section we shall establish several symmetry results for special Weingarten surfaces arising from the symmetry of the boundary. Some of them are not known even in the constant mean curvature case. Others are generalizations of well-know results for $H=$ const or simple extensions of results obtained recently in euclidean space.

Let $C_{1}, C_{2}$ be two circles in $\mathbf{H}^{3}$ with the same radius. Suppose $C_{1} \cup C_{2}$ is invariant under a rotation; that is, $C_{1}, C_{2}$ are on the same cylinder $C$. We will denote by $\operatorname{int}(C)$ the component of $\mathbf{H}^{3}-C$ containing the axis of $C$. The distance $d\left(C_{1}, C_{2}\right)$ between $C_{1}$ and $C_{2}$ is the distance between the "parallel" geodesic planes determined by $C_{1}$ and $C_{2}$.

It is well-known (see [11]) that if $C_{1} \cup C_{2}$ is the boundary of a minimal surface of revolution then $d\left(C_{1}, C_{2}\right) \leq 2 d_{0}$, where $d_{0}$ is the maximum value of the function $x\left(y_{0}\right)$ given by

$$
x\left(y_{0}\right)=\int_{y_{0}}^{+\infty} \frac{\sinh y_{0} \cosh y_{0}}{\cosh y} \sqrt{\frac{1}{\sinh ^{2} y \cosh ^{2} y-\sinh ^{2} y_{0} \cosh ^{2} y_{0}}} d y .
$$

Clearly the above function is bounded, and numerical computation shows that $d_{0} \approx 0.5$. (The above formula follows from the first integral of the second order differential equation satisfied by the generating curve [11].)

From now on, we will denote by $d_{0}$ the positive real number defined above.

We shall need the following lemma:

Lemma 3. Let $M$ be a compact connected embedded special Weingarten surface in $\mathbf{H}^{3}$ satisfying (1) for $f$ elliptic. Assume $\partial M$ is the union of two distinct circles $C_{1}, C_{2}$ lying in a cylinder $C$ of axis $\gamma$. Then, if $M \subset \overline{\operatorname{int}(C)}$ and $M \cap C=\partial M, M$ has a plane of symmetry.

Moreover, if $f$ does not change sign and if $d\left(C_{1}, C_{2}\right) \geq 2 d_{0}$, then $M$ lies between the parallel planes containing $C_{1}$ and $C_{2}$.

Proof. We denote by $P$ the plane of symmetry of $C_{1} \cup C_{2}$, that is, the plane such that $C_{1}$ is the symmetric image of $C_{2}$ with respect to $P$. We will prove $P$ is a plane of symmetry of $M$ as follows: We start by moving $P$ along $\gamma$ by doing hyperbolic translations. This movement gives rise to a 1-parameter family $\left\{P_{t}\right\}$ of 
geodesic planes starting from $P=P_{0}$ and cutting $\gamma$ orthogonally. We may choose a parameter $t$ such that $t$ is the oriented distance between $P_{t}$ and $P$. We claim that our assumptions in the first statement allow us to apply Alexandrov reflection, by the means of the family $\left\{P_{t}\right\}$, to conclude that $M$ inherits the symmetry of $\partial M$. Actually, to explain how to do this, we will denote by $M_{t}{ }^{*}$ the reflection on $P_{t}$ for $t \neq 0$ of the components of $M-P_{t}$ lying in the connected component of $\mathbf{H}^{3}-P_{t}$ not containing $P$. We begin the standard procedure by moving $P$ until $P_{t}$ is disjoint of $M$, i.e., $P_{t} \cap M=\emptyset$. Then moving $P_{t}$ back towards $P$, doing Alexandrov reflection during this movement, we find a first point of contact between $M$ and $M_{t}{ }^{*}$. Since $C$ is invariant under reflection on $P_{t}$, then, if by absurd $P_{t} \neq P$, this first point of contact cannot occurs at a boundary point of $\partial M$, because $\partial M \subset C$ and $M \cap C=\partial M$. So, if $t \neq 0$ we get a tangent point of contact, and both $M$ and $M_{t}{ }^{*}$ have the correct orientation at this point. We arrive to a contradiction, for the maximum principle says that $P_{t}, t \neq 0$, is a plane of symmetry of $M$. Then $P_{t}=P$, that is, $P$ is a plane of symmetry of $M$, as required.

To prove the second statement we will proceed as follows. Let $P_{d_{1}}$ and $P_{-d_{1}}$, $d_{1} \geq d_{0}$, be the geodesic planes passing through $C_{1}$ and $C_{2}$. Denote by $\mathcal{H}$ the region bounded by $P_{d_{1}}$ and $P_{-d_{1}}$, and by $P_{t}^{+}$the connected component of $\mathbf{H}^{3}-P_{t}$ not containing $P$. Under those conventions, notice that $M \cup\left(P_{d_{1}}^{+} \cap C\right) \cup\left(P_{-d_{1}}^{+} \cap C\right)$ is the boundary of a region $V$ in $\mathbf{H}^{3} ; V$ is contained in $\overline{\operatorname{int}(C)}$, and $\partial V$ is not smooth over $\partial M$. Note that, since $f$ does not change sign, either the mean curvature vector $\vec{H}$ of $M$ is pointing into the interior of $V$ or else $\vec{H}$ is pointing towards the exterior of $V$. We claim $\vec{H}$ is an inward pointing normal vector. Indeed, set $p=\gamma \cap P$ and let $\alpha \subset P$ be a geodesic line cutting $\gamma$ orthogonally at $p$. Let $\left\{\zeta_{t}\right\}$ be the 1-parameter family of minimal surfaces of revolution with axis $\gamma$ and generating curve $c_{t}$ such that $\alpha$ is the symmetry line of $c_{t}$ and $t=d\left(\zeta_{t}, \gamma\right)$. Note that, as $d\left(C_{1}, C_{2}\right) \geq 2 d_{0}$, the family $\left\{\zeta_{t}\right\}$ is inside $\mathcal{H}$. To prove the claim, we move $\zeta_{t}$ from infinity towards $M$, making $t \rightarrow 0$, to reach a first interior point of contact. By the comparison principle $\vec{H}$ points into $V$, as required. To conclude the proof, if $M \cap \operatorname{ext}(\mathcal{H}) \neq \emptyset$, one derives a contradiction by the same argument as in the preceding paragraph, making use of the family $\left\{P_{t}\right\}$ coming from infinity towards $\partial \mathcal{H}$.

We remark that there always exists an umbilic surface in the class of any Weingarten surface satisfying (1) for $f$ elliptic: If $f(0)=0$, any geodesic plane is in the class. If $f^{2}(0)=1$, any horosphere is in the class. If $f^{2}(0)<1$, any equidistant surface with mean curvature $f(0)$ is in the class. If $f^{2}(0)>1$, any sphere of radius $\rho$ with coth $\rho=|f(0)|$ is in the same class of $M$.

Theorem 1. Let $M$ be a connected properly embedded special Weingarten surface in $\mathbf{H}^{3}$ satisfying (1) for $f$ elliptic. Assume $\partial M \neq \emptyset$. Suppose $f^{2} \leq 1$. We have the following:

(1) If $\partial_{\infty} M=\emptyset$ and $\partial M$ is a circle, then $M$ is totally umbilic.

(2) Assume that $M$ is compact and $\partial M$ is the union of two circles $C_{1}, C_{2}$ of the same radius with $C_{1} \cup C_{2}$ invariant under a rotation. If $d\left(C_{1}, C_{2}\right) \geq 2 d_{0}$, $|f(0)|=1$, and $f$ does not change sign, then $M$ is part of a complete embedded special surface of revolution. Furthermore, the generating curve attains one and only one global minimum, and each end is asymptotically umbilic in the sense of Theorem 2, section 4 .

Proof of Statement 1. We suppose $\partial_{\infty} M=\emptyset$, and then $M$ is compact. 
Let $P$ be the geodesic plane in $\mathbf{H}^{3}$ containing $\partial M$. We consider the halfspace model $\mathbf{H}^{3}=\{(x, y, z), z>0\}$. Without loss of generality, we can suppose $P=\{x=$ $0\}$. Let $H_{0}$ and $H_{0}{ }^{*}$ be the unique horospheres in $\mathbf{H}^{3}$ such that $H_{0} \cap P=H_{0}{ }^{*} \cap P=$ $\partial M$. Denote by $D_{0}, D_{0}{ }^{*}, D_{0} \subset H_{0}, D_{0}{ }^{*} \subset H_{0}{ }^{*}$, the compact pieces with boundary $\partial M$. It follows from [2] that $M$ is inside the region of $\mathbf{H}^{3}$ with boundary $D_{0} \cup D_{0}{ }^{*}$. Now, we claim $M$ is contained in one side of $P$. Indeed, if $f(0)=0$, the maximum principle shows that $M$ is the geodesic disk $D \subset P$ whose boundary is equal to $\partial M$. But if $f(0) \neq 0$, we will arrive to a contradiction by the following argument. Take the family $\{S\}$ of umbilic surfaces contained in $\mathbf{H}^{3}-P$ which is in the same class of $M$, i.e., $H=f(0)$, by choosing an appropriate normal field $N$. We also require that the mean curvature vector of each surface $S$ points into the region of $\mathbf{H}^{3}-S$ not containing $P$ (this makes sense since $f(0) \neq 0$ ). Now it is clear that if $M \cap\{x<0\} \neq \emptyset$ and $M \cap\{x>0\} \neq \emptyset$, we may compare of $M$ with a member $S$ of the family in a suitable way to obtain a contradiction by the maximum principle. This proves the previous claim, and we conclude $M$ is in one side of $P$. Finally, the standard Alexandrov principle, using the family of geodesic planes orthogonal to $P$, allows us to conclude that $M$ inherits the symmetry of $\partial M$, i.e., $M$ is invariant under a 1-parameter group of rotations. Clearly, the curvature lines are meridians and parallels. Note that either $M$ is totally umbilic or else the singularities of the lines of curvature are isolated, by Bryant's theorem (see Proposition 2). Also note that the only possible singularities of the lines of curvatures are the points on the rotation axis, and there the singularity has nonnegative index. Consequently $M$ is totally umbilic.

Proof of Statement 2. Let $C$ be the cylinder with axis $\gamma$ containing $C_{1} \cup C_{2}$. Let $\left\{C_{t}\right\}, t \geq 0$, be the 1-parameter family of cylinders with the same axis $\gamma$, with $t=d\left(C_{t}, \gamma\right)$. We assert that $M \subset \overline{\operatorname{int}(C)}$ and $M \cap C=\partial M$. Indeed, if this is not the case, then as $t \rightarrow+\infty$ one may find a "last" cylinder $C_{t_{1}}$ such that $C_{t_{1}} \cap M \neq \emptyset$ and $C_{t} \cap M=\emptyset$ for $t>t_{1}$. If $M \cap\left(\mathbf{H}^{3}-\overline{\operatorname{int}(C)}\right) \neq \emptyset$, then $t_{1}>0$; hence we get a contradiction with the standard comparison principle (because the mean curvature of $M$ is not greater than 1). For the same reasons, $M \cap C=\partial M$. It can be inferred from a straightforward application of the Alexandrov principle and Lemma 3 that $M$ is a surface of revolution whose generating curve attains a local minimum. Suppose without loss of generality that $\gamma$ is the $z$-axis (in the upper halfspace model). Assume $C_{1}, C_{2}$ belong to the horospheres $\Theta_{z_{1}}=\left\{z=z_{1}\right\}$ and $\Theta_{z_{2}}=\left\{z=z_{2}\right\}$, respectively, with $z_{1}<z_{2}$. Consider the 1-parameter family of horospheres $\Theta_{t}=\{z=t\}$ for $t>z_{2}$. Recall that we may move this family along $\gamma$ by making use of hyperbolic translations. Now, doing $t \downarrow z_{2}$ coming from infinity towards $M$, it follows from the maximum principle and from the proof of Lemma 3 that $M$ is entirely contained inside the region $\left\{z \leq z_{2}\right\}$ of $\mathbf{H}^{3}$. By taking Lemma 3 into account again, one may conclude that $M$ is still inside the region $\left\{z \geq z_{1}\right\}$. Finally, doing Alexandrov reflection, one may derive our previous claim, as desired. Recall that it follows from the proof of Lemma 3 that $\vec{H}$ is an inward pointing normal vector. Thus we have $H=f \leq 0$ on $M$ with respect to the outward normal orientation. Therefore we are able to apply Theorem 2 , section 4 , to conclude that the generating curve attains one and only one global minimum since $f(0)=-1$. Furthermore in Theorem 2, section 4, it is proved that each end of $M$ is asymptotically umbilic. This concludes the proof of Theorem 1. 
Remark 2. Statement 1 of Theorem 1 when $H=$ const and $H^{2} \leq 1$ was obtained in [14]; the sharp result (assuming $M$ immersed) followed in [2]. The general situation for $H=$ const, $H^{2}>1$, as in the euclidean case, is still not known. Partial results were obtained in [2]. See also J. L. Barbosa and R. Sa Earp, Prescribed mean curvature hypersurfaces in $H^{n+1}$ with convex planar boundary. II, Séminaire de Théorie Spectrale et Géométrie, Vol. 16, Univ. Grenoble, 1998, pp. 43-79. CMP 99:07.

The following corollary is immediate:

Corollary 1. Let $M$ be a compact embedded surface in $\mathbf{H}^{3}$ with constant mean curvature 1. Suppose $\partial M$ is the union of two circles $C_{1}, C_{2}$ of the same radius, with $C_{1} \cup C_{2}$ invariant under a rotation.

If $d\left(C_{1}, C_{2}\right) \geq 2 d_{0}$, then $M$ is a piece of a Catenoid Cousin.

Proposition 3. Let $M$ be a compact embedded special Weingarten surface in $\mathbf{H}^{3}$ satistying (1) for $f$ elliptic. Assume there exists a cylinder $C$ such that $\partial M \subset C$ and $M \subset \overline{\operatorname{int}(C)}$. Suppose $f$ satisfies, $f<0, f(t)+\sqrt{t+1}$ is an increasing function for $t \geq 0$, and $f(c)+\sqrt{c+1}=0$, where $H=-\sqrt{c+1}$ is the mean curvature of $C$ with respect to the outward normal orientation (that is, $C$ is $f$-special; see Remark 4).

Then if $\partial M$ is the union of two circles $C_{1}, C_{2}$ invariant under the group of rotations of $C$, and if $d\left(C_{1}, C_{2}\right) \geq 2 d_{0}, M$ is part of a complete embedded periodic special surface of revolution.

Proof. Let us consider the half-space model of $\mathbf{H}^{3}$. First, notice that our assumptions imply that $C$ is the unique cylinder in the same class of $M$ i.e., $C$ satisfies the Weingarten relation (1). Thus, ellipticity yields $M \cap C=\partial M$. Since our hypotheses imply $f(0)<-1$, we may use spheres of the same class as $M$, above $M$, to show as in Theorem 1 that $M$ lies between the parallel euclidean planes containing $C_{1}$ and $C_{2}$ respectively (which are hyperbolic horospheres, denoted by $\Theta_{z_{1}}$ and $\Theta_{z_{2}}$ in the proof of Theorem 1). So we may apply Lemma 3 and the maximum principle to show as in statement 2 of Theorem 1, that $M$ is invariant under the group of rotations keeping $C$ invariant. Furthermore, since Lemma 3 shows that $M$ has a plane of symmetry, we deduce that the graph generating $M$ has a local minimum. By Remark 6 , section 4 , we conclude $M$ is part of a complete embedded periodic special surface of revolution.

To conclude this section we shall figure out a characterization of geodesic disks of spheres proved by Braga Brito and the first author in [3], when the ambient space is euclidean space. The proof is the same in both euclidean and hyperbolic space, so we will omit it here. Let $M$ be an immersed disk type special Weingarten surface in $\mathbf{H}^{3}$ satisfying (1) for $f$ elliptic. Assume $\partial M$ is a circle of radius $\rho$. Suppose $f$ is analytic with $f(0)>0$. Then:

a) $f(0) \leq \operatorname{coth} \rho$.

b) If $f(0)=\operatorname{coth} \rho, M$ is a half sphere.

Remark 3. There is another characterization with same statement and proof in both euclidean and hyperbolic space derived in [3]: If $M$ is a disk type embedded special Weingarten surface in $\mathbf{H}^{3}$ satisfying (1) for $f$ elliptic, if $\partial M$ is a circle of radius $\rho$, if $f$ is positive $f>0$, and if $M$ cuts transversely, along $\partial M$, the geodesic plane $P$ containing $\partial M$, then $M$ is a geodesic disk of a sphere. 


\section{Rotational SPecial Weingarten SuRfaces. EXISTENCE AND UNIQUENESS}

In this section, we are interested in special Weingarten surfaces of revolution in the hyperbolic space $\mathbf{H}^{3}$. We choose the model of the 3-ball; hence our surfaces are generated by a curve of the 2-hyperbolic disc $\mathbf{D}=\left\{(u, v) \in \mathbf{R}^{2} \mid u^{2}+v^{2}<1\right\}$. We choose the following coordinates $(x, y), x, y \in \mathbf{R}$, of $\mathbf{D}$ : Let $p \in \mathbf{D}$, and let $\gamma$ be the unique geodesic passing through $p$ and orthogonal to the horizontal geodesic $\{v=0\}$. Let $q$ be the intersection between the two geodesics. Then $x$ is equal to the oriented hyperbolic distance between $q$ and 0 , and $y$ is the oriented hyperbolic length of $\gamma$ between $p$ and $q$. This means that $x \geq 0$ (resp. $y \geq 0$ ) if and only if $u \geq 0$ (resp. $v \geq 0$ ), where $(u, v)$ are the euclidean coordinates of $p$. Note that the coordinate curves $\{x=$ const $\}$ are the geodesics orthogonal to the $u$-axis and $\left\{y=y_{0}\right\}$ are the equidistant-curves of the $u$-axis, namely the arcs of circles passing through the points $(-1,0)$ and $(1,0)$ making an angle $\alpha$ with $\partial \mathbf{D}$ such that $\cos (\alpha)=\tanh \left(y_{0}\right)$. It is well-known that the metric of $\mathbf{D}$ with $(x, y)$-coordinates is

$$
d s^{2}=\cosh ^{2}(y) d x^{2}+d y^{2} .
$$

Now, let $\gamma \subset \mathbf{D}$ be a curve and let us call $M$ the revolution surface generated by $\gamma$, where the revolution axis is always the $x$-axis (which is also the $u$-axis). Suppose that $\gamma$ is the graph of a positive $C^{2}$ function $y=y(x)$. By the outward normal orientation on $M$ we mean the orientation given on $\gamma$ by the unit normal field pointing toward the direction of increasing $y$. Straightforward computations show that the principal curvatures of $M$ with respect to the outward normal orientation are

$$
\begin{aligned}
& \lambda_{1}(x)=\frac{y^{\prime \prime} \cosh (y)-2 \sinh (y) y^{\prime 2}-\sinh (y) \cosh ^{2}(y)}{\left(\cosh ^{2}(y)+y^{\prime 2}\right)^{3 / 2}}, \\
& \lambda_{2}(x)=-\frac{\cosh ^{2}(y)}{\sinh (y)\left(\cosh ^{2}(y)+y^{\prime 2}\right)^{1 / 2}} .
\end{aligned}
$$

Note that $\lambda_{1}(x)$ is the hyperbolic curvature of $\gamma$ as a planar curve. From now on we will always assume that $f$ is an elliptic function (see equation (2)). Let $M$ be a surface of $\mathbf{H}^{3}$ and let $N$ be a normal unit field over $M$. Recall that $M$ is an $f$-surface (with respect to $N$ ) if and only if the principal curvatures of $M$ satisfy the relation (see equation (1)):

$$
\frac{\lambda_{1}+\lambda_{2}}{2}(N)=f\left(\left[\frac{\lambda_{1}-\lambda_{2}}{2}\right]^{2}\right) .
$$

Recall that for every elliptic function $f$ any totally umbilic hypersurfaces with mean curvature $f(0)$ are $f$-surfaces. Namely, if $|f(0)|>1$ they are the compact spheres of hyperbolic radius $r$ with $\operatorname{coth}(r)=|f(0)|$, and if $|f(0)| \leq 1$ they are the intersections of spheres with the 3 -ball which intersect the boundary $\partial \mathbf{H}^{3}$ at an angle $\alpha$ such that $\cos (\alpha)=f(0)$.

Now, let $M$ be a surface of revolution of $\mathbf{H}^{3}$ and let $\gamma$ be the plane curve generating $M$. Let us assume that $\gamma$ is the graph of a positive $C^{2}$ function $y$. Then $M$ 
is an $f$-surface if and only if $f$ satisfies the relation $F\left(y, y^{\prime}, y^{\prime \prime}\right)=0$, where

$$
\begin{aligned}
& F\left(y, y^{\prime}, y^{\prime \prime}\right) \\
& =\frac{y^{\prime \prime} \cosh (y) \sinh (y)-\left(2 \sinh ^{2}(y)+\cosh ^{2}(y)\right) y^{2}-\cosh ^{2}(y)\left(\sinh ^{2}(y)+\cosh ^{2}(y)\right)}{2 \sinh (y)\left(\cosh ^{2}(y)+y^{\prime 2}\right)^{3 / 2}} \\
& -f\left(\left[\frac{y^{\prime \prime} \cosh (y) \sinh (y)+\left(1-\sinh ^{2}(y)\right) y^{\prime 2}+\cosh ^{2}(y)}{2 \sinh (y)\left(\cosh ^{2}(y)+y^{\prime 2}\right)^{3 / 2}}\right]^{2}\right) .
\end{aligned}
$$

From the ellipticity of $f$, we deduce that $\partial F / \partial y^{\prime \prime}>0$, so $F$ is a strictly increasing function of the third variable.

Proposition 4. Let $f$ be an elliptic function. Then there exists an $f$-surface of revolution, possibly noncomplete, if and only if $f$ satisfies the following condition:

$$
\lim _{t \rightarrow+\infty}\left(t-f\left(t^{2}\right)\right)>0
$$

Proof. Suppose there exists an $f$-surface of revolution $M$. We can assume that part of $M$ is generated by the graph of a strictly positive function $y(x)$. It follows that there exist real numbers $y_{0}>0, y_{0}^{\prime}$ and $y_{0}^{\prime \prime}$ such that $F\left(y_{0}, y_{0}^{\prime}, y_{0}^{\prime \prime}\right)=0$. But this is equivalent to

$$
t-f\left(t^{2}\right)=\frac{\cosh ^{2}\left(y_{0}\right)}{\sinh \left(y_{0}\right)\left(\cosh ^{2}\left(y_{0}\right)+y_{0}^{\prime 2}\right)^{1 / 2}}
$$

where

$$
t=\frac{y_{0}^{\prime \prime} \cosh \left(y_{0}\right) \sinh \left(y_{0}\right)+\left(1-\sinh ^{2}\left(y_{0}\right)\right) y_{0}^{\prime 2}+\cosh ^{2}\left(y_{0}\right)}{2 \sinh \left(y_{0}\right)\left(\cosh ^{2}\left(y_{0}\right)+y_{0}^{\prime 2}\right)^{3 / 2}} .
$$

As $f$ is elliptic, we know that $\left(t-f\left(t^{2}\right)\right)$ is a strictly increasing function. Hence

$$
\lim _{t \rightarrow+\infty}\left(t-f\left(t^{2}\right)\right)>\frac{\cosh ^{2}\left(y_{0}\right)}{\sinh \left(y_{0}\right)\left(\cosh ^{2}\left(y_{0}\right)+y_{0}^{\prime 2}\right)^{1 / 2}},
$$

and so $\lim _{t \rightarrow+\infty}\left(t-f\left(t^{2}\right)\right)>0$.

Conversely, assume that $\lim _{t \rightarrow+\infty}\left(t-f\left(t^{2}\right)\right)>0$. Note that

$$
\lim _{t \rightarrow-\infty}\left(t-f\left(t^{2}\right)\right)<\lim _{t \rightarrow+\infty}\left(t-f\left(t^{2}\right)\right)
$$

Clearly, there exist strictly positive real numbers $y_{0}, y_{0}^{\prime}>0$ such that

$$
\lim _{t \rightarrow-\infty}\left(t-f\left(t^{2}\right)\right)<\frac{\cosh ^{2}\left(y_{0}\right)}{\sinh \left(y_{0}\right)\left(\cosh ^{2}\left(y_{0}\right)+y_{0}^{\prime 2}\right)^{1 / 2}}<\lim _{t \rightarrow+\infty}\left(t-f\left(t^{2}\right)\right) .
$$

On the other hand, $F\left(y_{0}, y_{0}^{\prime},.\right)$ is an increasing function. Therefore, we conclude from the ellipticity that

$$
\lim _{s \rightarrow-\infty} F\left(y_{0}, y_{0}^{\prime}, s\right)<0 \text { and } \lim _{s \rightarrow+\infty} F\left(y_{0}, y_{0}^{\prime}, s\right)>0 .
$$

This let us conclude that there exists a real number $y_{0}^{\prime \prime}$ such that $F\left(y_{0}, y_{0}^{\prime}, y_{0}^{\prime \prime}\right)=0$. Then, using the implicit function theorem, we can prove that there exists a real $C^{1}$ function $h$, defined on a neighborhood of $\left(y_{0}, y_{0}^{\prime}\right)$, satisfying

$$
F\left(y, y^{\prime}, y^{\prime \prime}\right)=0 \Leftrightarrow y^{\prime \prime}=h\left(y, y^{\prime}\right), \quad \text { and } \quad h\left(y_{0}, y_{0}^{\prime}\right)=y_{0}^{\prime \prime} .
$$

Now Picard's theorem shows that there exists a unique function $y(x)$ satisfying the last ODE with the initial conditions $y(0)=y_{0}$ and $y^{\prime}(0)=y_{0}^{\prime}$. Clearly, the graph of this function generates an $f$-surface, as desired. 
From now on, we only consider the outward normal orientation for a graph of D, and we will always suppose that $f(0) \leq 0$. For later use, we state the following lemmas.

Lemma 4. Let $f$ be an elliptic function and let $y$ be a $C^{2}$ real function whose graph $\gamma$ generates an $f$-surface $M$. Suppose that $y^{\prime}\left(x_{0}\right)=0$ for a real number $x_{0}$. Then $\gamma$ is symmetric with respect to the geodesic orthogonal to $\gamma$ at the point $\left(x_{0}, y\left(x_{0}\right)\right.$ ) (hence $M$ is symmetric with respect to the totally geodesic hyperplane of $\mathbf{H}^{3}$ orthogonal to $\mathbf{D}$ which contains this geodesic).

Proof. Observe that the functions $y$ and $z(x)=y\left(2 x_{0}-x\right)$ satisfy the same second order differential equation with the same initial conditions at $x_{0}$, since $y\left(x_{0}\right)=$ $z\left(x_{0}\right)$ and $y^{\prime}\left(x_{0}\right)=0=z^{\prime}\left(x_{0}\right)$. Actually (1) can be written locally in the form $y^{\prime \prime}=h\left(y, y^{\prime}\right)$, see the proof of Proposition 4. So we have $y=z$.

Lemma 5. Let $f$ be an elliptic function and let y be a $C^{2}$ real function whose graph $\gamma$ generates an $f$-surface $M$. Suppose that $M$ has an umbilic point. Then $M$ is totally umbilic.

Proof. This is a direct consequence of Proposition 2, section 2. If $M$ admits an umbilic point outside the revolution axis, then $M$ has at least a circle of umbilic points. Since Proposition 2, section 2, shows that either $M$ is totally umbilic or umbilic points are isolated, we conclude in this case that $M$ is totally umbilic. If the umbilic point stays on the $x$-axis, note that the index of this umbilic point (if it is isolated) is +1 , but Proposition 2, section 2, shows that this index should be negative and we again conclude that $M$ is totally umbilic.

We remark that we can give a direct proof of Lemma 5. For this, it suffices to show that there is a $C^{2}$ curve tangent to $\gamma$ at the umbilic point generating a totally umbilic surface with constant mean curvature $f(0)$ with respect to the outward normal orientation.

Lemma 6. Let $f$ be an elliptic function and let $y$ be a $C^{2}$ positive monotone function whose graph generates a non-totally-umbilic $f$-surface $M$ of $\mathbf{H}^{3}$. Then

$$
\lambda_{2}^{\prime}=y^{\prime} \operatorname{coth}(y)\left(\lambda_{1}-\lambda_{2}\right) .
$$

Consequently, the principal curvatures of $M$ also are monotone and $\lambda_{1}$ is increasing if and only if $\lambda_{2}$ is decreasing.

Proof. A simple computation shows that the principal curvatures of $M$ satisfy the formula in the statement. Then, Lemma 5 shows that $\lambda_{2}$ is a monotone function. Moreover, by differentiating the relation (1) we obtain

$$
\lambda_{1}^{\prime}+\lambda_{2}^{\prime}=\left(\lambda_{1}^{\prime}-\lambda_{2}^{\prime}\right) \cdot\left(\lambda_{1}-\lambda_{2}\right) \cdot f^{\prime}\left(\left[\frac{\lambda_{1}-\lambda_{2}}{2}\right]^{2}\right) .
$$

Using the ellipticity of $f$, we infer that

$$
\left|\lambda_{1}^{\prime}+\lambda_{2}^{\prime}\right|<\left|\lambda_{1}^{\prime}-\lambda_{2}^{\prime}\right|
$$

which finishes the proof.

Now, recall that a cylinder of $\mathbf{H}^{3}$ is a complete surface of revolution generated by the graph of a constant function $y(x)=\tau>0$, which will be denoted by $C_{\tau}$. 
Note that the principal curvatures of $C_{\tau}$ are $\lambda_{1}=-\tanh (\tau)$ and $\lambda_{2}=-\operatorname{coth}(\tau)$. Then, if $f$ is an elliptic function, the cylinder $C_{\tau}$ is a $f$-surface if and only if

$$
\frac{\tanh (\tau)+\operatorname{coth}(\tau)}{2}+f\left(\left[\frac{\tanh (\tau)-\operatorname{coth}(\tau)}{2}\right]^{2}\right)=0 .
$$

Proposition 5. Let $f$ be an elliptic function with $f^{\prime} \geq 0$. Then there exists an $f$-special cylinder if and only if $f(0)<-1$. Furthermore, this cylinder is unique.

Proof. As $f$ is elliptic, the function $t+f\left(t^{2}\right)$ is increasing. Moreover, as $f$ is increasing, the function

$$
g(t)=\frac{\tanh (t)+\operatorname{coth}(t)}{2}+f\left(\left[\frac{\tanh (t)-\operatorname{coth}(t)}{2}\right]^{2}\right)
$$

is decreasing. Let us assume first that $f(0)<-1$. Note that $\lim _{t \rightarrow+\infty} g(t)=$ $1+f(0)<0$ and $\lim _{t \rightarrow 0} g(t)=\lim _{t \rightarrow+\infty}\left(t+f\left(t^{2}\right)\right)=+\infty$. So, there exists a unique positive real number $\tau$ satisfying $g(\tau)=0$, i.e., there exists a unique $f$ special cylinder.

Conversely, suppose there exists an $f$-special cylinder $C_{\tau}$. Consequently $g(\tau)=$ 0 , and we deduce that (as $f$ is increasing)

$$
f(0) \leq-\frac{\tanh (\tau)+\operatorname{coth}(\tau)}{2}<-1 .
$$

As before, we may conclude that $C_{\tau}$ is the unique $f$-special cylinder.

Remark 4. Note that in the euclidean case ellipticity of $f$ ensures uniqueness of the $f$-special cylinder (see [18]). In the hyperbolic case, if we do not assume $f$ increasing, there may exist many, even infinitely many, $f$-special cylinders. To see this, set

$$
X=X(t)=\left[\frac{\tanh (t)-\operatorname{coth}(t)}{2}\right]^{2} .
$$

We then have $g(t)=h(X)=\sqrt{X+1}+f(X)$ with $X \in[0,+\infty[$. Observe that each zero of $h$ gives an $f$-special cylinder. Moreover, we can write $h$ as an integral:

$$
h(X)=\int_{0}^{X}\left(f^{\prime}(t)+\frac{1}{2 \sqrt{t+1}}\right) d t+f(0)+1 .
$$

Then

$$
h(X)=0 \Leftrightarrow \int_{0}^{X}\left(f^{\prime}(t)+\frac{1}{2 \sqrt{t+1}}\right) d t=-(f(0)+1) .
$$

Now observe that the last integral is the (oriented) area of the finite plane region limited by the graph of the two functions $f^{\prime}(t)$ and $-\frac{1}{2 \sqrt{t+1}}$ for $t$ between 0 and $X$. Also, $f$ elliptic means only that the graph of $f^{\prime}$ stays between the graph of the two functions $\frac{1}{2 \sqrt{t}}$ and $-\frac{1}{2 \sqrt{t}}$. So it is not difficult to find elliptic functions $f$ such that the associated function $h$ has as many zeros as we want (even infinitely many). Finally, observe that the weaker condition $f^{\prime}(t) \geq-\frac{1}{2 \sqrt{t+1}}$ ensures uniqueness of the $f$-special cylinder, since the function $h$ is increasing.

Theorem 2. Let $f$ be an elliptic function with $f(0) \leq 0$ and $\lim _{t \rightarrow+\infty}\left(t+f\left(t^{2}\right)\right)>$ 0 . Let $\tau>0$ be a positive real number satisfying

$$
\frac{\tanh (\tau)+\operatorname{coth}(\tau)}{2}+f\left(\left[\frac{\tanh (\tau)-\operatorname{coth}(\tau)}{2}\right]^{2}\right)>0,
$$




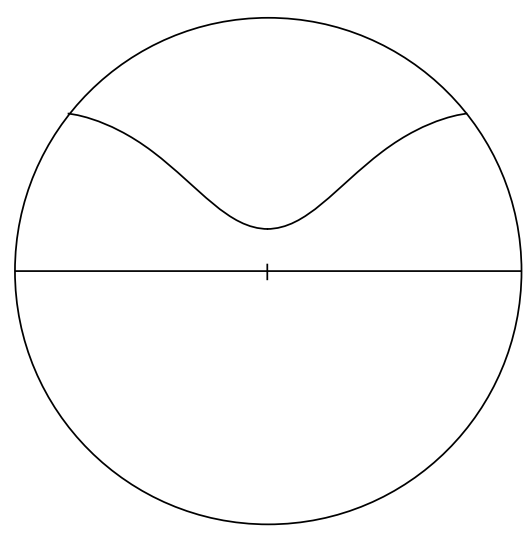

FIGURE 1

$$
\operatorname{coth}(\tau)<\lim _{t \rightarrow+\infty}\left(t-f\left(t^{2}\right)\right)
$$

Then there exists a unique complete curve $\gamma_{\tau}$ which is the graph of a $C^{2}$ function $y_{\tau}(x)$, admitting a minimum at $(0, \tau)$, symmetric with respect to the $y$-axis and generating a complete embedded $f$-surface of revolution $M_{\tau}$.

Moreover, if $f^{\prime} \geq 0$, the curve $\gamma_{\tau}$ has the following behaviour.

1) If $-1<f(0) \leq 0$, then $y_{\tau}$ is defined on an open interval $]-x_{\tau}, x_{\tau}[$ with $0<x_{\tau}<+\infty ; y_{r}$ is increasing on $\left[0, x_{\tau}\right.$ [ and satisfies $\lim _{x \rightarrow x_{\tau}} y_{\tau}=+\infty$. Also $\gamma_{\tau}$ has a limit direction tangent and makes an angle $\left.\left.\theta \in\right] 0, \pi / 2\right]$ with $\partial \mathbf{D}$ such that $\cos (\theta)=|f(0)|$ (see figure 1). Furthermore, both the principal curvatures of $M_{\tau}$ go to $f(0)$ when $x$ goes to $\pm x_{\tau}$. Consequently $M_{\tau}$ is asymptotically umbilic.

2) If $f(0)=-1$, then $y_{\tau}$ is defined on $]-\infty,+\infty[$, is increasing on $[0,+\infty[$ and satisfies $\lim _{x \rightarrow \infty} y_{\tau}=+\infty$. Also $\gamma_{\tau}$ has a limit tangent direction and is tangent to $\partial \mathbf{D}$ (see figure 2). Furthermore, both principal curvatures of $M_{\tau}$ go to -1 when $x$ goes to $\pm \infty$. It follows that $M_{\tau}$ is asymptotically umbilic.

3) If $f(0)<-1$, then $y_{\tau}$ is defined on $]-\infty,+\infty[$ and is periodic (see figure 3 ).

In the same way, if $-1 \leq f \leq 0$ (without supposing $f^{\prime} \geq 0$ ) then cases 1) and 2) still hold.

Remark 5. (a) Note that in each case $\gamma_{\tau}$ has the same geometrical behaviour as the curve generating a complete embedded surface of $\mathbf{H}^{3}$ with constant mean curvature $f(0)$.

(b) We deduce that if $-1<f(0) \leq 0$ and $f^{\prime} \geq 0$ (or $-1 \leq f \leq 0$ ), then each end of the $f$-surfaces given by Theorem 2 is $C^{1}$ asymptotic to an end of a totally umbilic surface of $\mathbf{H}^{3}$ with constant mean curvature $f(0)$.

Proof of Theorem 2. We have seen that the graph of a positive function $y_{\tau}$ generates an $f$-surface if and only if $F\left(y_{\tau}, y_{\tau}^{\prime}, y_{\tau}^{\prime \prime}\right)=0$. Also, $f$ elliptic implies that $F$ is strictly increasing with respect to $y_{\tau}^{\prime \prime}$.

A computation shows that

$$
F(\tau, 0,0)=-\frac{\tanh (\tau)+\operatorname{coth}(\tau)}{2}-f\left(\left[\frac{\tanh (\tau)-\operatorname{coth}(\tau)}{2}\right]^{2}\right)<0,
$$




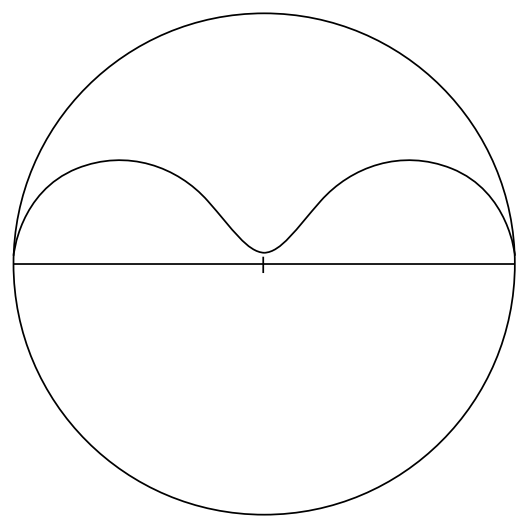

Figure 2

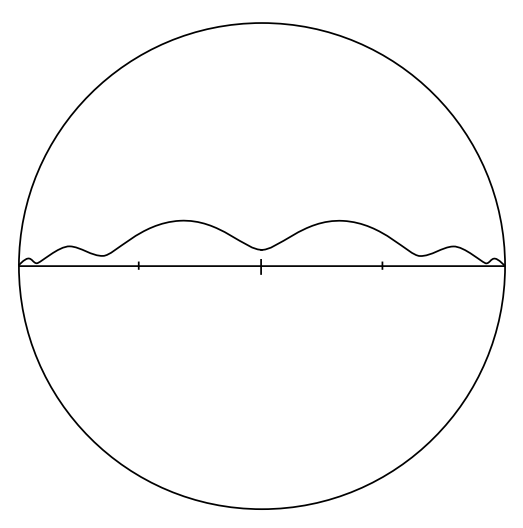

FiguRE 3

and

$$
\lim _{t \rightarrow+\infty} F(\tau, 0, t)=\lim _{t \rightarrow+\infty}\left(t-f\left(t^{2}\right)\right)-\operatorname{coth}(\tau)>0 .
$$

Therefore, there is an unique positive real number $y_{0}^{\prime \prime}>0$ satisfying $F\left(\tau, 0, y_{0} "\right)=$ 0 . Now, the implicit function theorem shows that there exists a $C^{1}$ real function $h$ defined in a neighborhood of $(\tau, 0)$ satisfying

$$
F\left(y_{\tau}, y_{\tau}^{\prime}, y_{\tau}^{\prime \prime}\right)=0 \Leftrightarrow y_{\tau}^{\prime \prime}=h\left(y_{\tau}, y_{\tau}^{\prime}\right), \quad \text { and } \quad h(\tau, 0)=y_{0}^{\prime \prime} .
$$

Furthermore, Picard's theorem shows that the above differential equation has a unique solution $y_{\tau}$ satisfying $y_{\tau}(0)=\tau$ and $y_{\tau}^{\prime}(0)=0$. Lemma 4 shows that $y_{\tau}(-x)=y_{\tau}(x)$, so we may suppose $y_{\tau}$ is defined on an interval $]-x_{1}, x_{1}[$. If $x_{1}=+\infty$ we are done, so suppose $0<x_{1}<+\infty$. Note that $y_{\tau}^{\prime}(x)>0$ for $x>0$ near 0 . If $y_{\tau}^{\prime}$ had another zero after $0, y_{\tau}$ would have another symmetry; but then $y_{\tau}$ would be a periodic function, and its graph $\gamma_{\tau}$ would be complete, as we wish. So suppose that $y_{\tau}^{\prime}(x)>0$ for $x>0$; consequently $y_{\tau}(x)$ has a positive limit $y_{1}$ as $x$ goes to $x_{1}$. If $y_{1}=+\infty$ the graph $\gamma_{\tau}$ is complete, so suppose that $0<y_{1}<+\infty$. 
Observe that $\lambda_{2}(0)=-\operatorname{coth}(\tau)$ and

$$
\lambda_{1}(0)=\frac{y_{0}^{\prime \prime}}{\cosh ^{2}(\tau)}-\tanh (\tau),
$$

and so $\lambda_{2}(0)<\lambda_{1}(0)$. Since the $f$-surface generated by $\gamma_{\tau}$ is not totally umbilic, Lemma 5 shows that $\lambda_{2}(x)<\lambda_{1}(x)$ for every $x$. Moreover, Lemma 6 says that $\lambda_{2}(x)$ is strictly increasing and $\lambda_{1}(x)$ is strictly decreasing on $\left[0, x_{1}[\right.$, so they have finite limit when $x$ goes to $x_{1}$. Hence $y_{\tau}^{\prime}$ has a positive limit $y_{1}^{\prime}$.

Note also that, as $f$ is elliptic, the function

$$
G\left(\lambda_{1}, \lambda_{2}\right)=\frac{\lambda_{1}+\lambda_{2}}{2}-f\left(\left[\frac{\lambda_{1}-\lambda_{2}}{2}\right]^{2}\right)
$$

is strictly increasing with respect to $\lambda_{1}$ and $\lambda_{2}$, and $G(f(0), f(0))=0$. We get from this and from the above observations that $\lambda_{2}(x)<f(0)<\lambda_{1}(x)$. If $f(0)<0$ the first inequality shows that $y_{1}^{\prime}<+\infty$. If $f(0)=0$ we also have $y_{1}^{\prime}<+\infty$, as otherwise the graph $\gamma_{\tau}$ would be tangent and into one side of the geodesic $\left\{x=x_{1}\right\}$. However, those two curves generate two $f$-surfaces of revolution. This last situation contradicts the maximum principle with boundary.

Thus in all cases we have $y_{1}^{\prime}<+\infty$, and as $\lambda_{1}$ has a finite limit we derive that $y_{\tau}^{\prime \prime}$ also has a finite limit $y_{1}^{\prime \prime}$. As $F$ is a continuous function we have $F\left(y_{1}, y_{1}^{\prime}, y_{1}^{\prime \prime}\right)=0$. So, using the implicit function theorem as above, we can extend $y_{\tau}$ beyond $x_{1}$. Repeating this argument we see that we obtain a complete symmetric curve $\gamma_{\tau}$ generating an $f$-surface, which is the graph of a $C^{2}$ function $y_{\tau}$ defined on $]-x_{\tau}, x_{\tau}[$, $0<x_{\tau} \leq+\infty$, as claimed. This completes the proof of the first assertion.

Proof of Statement (3). Now let us assume $f^{\prime} \geq 0$. Suppose first that $f(0)<$ -1 . Note that in this case, we have $x_{\tau}=+\infty$. Otherwise, we could find an $f$ special sphere with mean curvature $f(0)$ tangent to and on one side of $M_{\tau}$, which contradicts the maximum principle. Then $y_{\tau}$ is defined for all real numbers $x \in \mathbf{R}$. Now we are going to show that $y_{\tau}$ is periodic. To the contrary, assume that $y_{\tau}$ is an increasing function on $[0,+\infty$ [ and consequently has a positive limit $c>0$ when $x$ goes to $+\infty$. The last argument with $f$-special spheres shows that $c \neq+\infty$. Hence, $0<c<+\infty$. Recall that $\lambda_{2}$ is strictly increasing and $\lambda_{1}$ is strictly decreasing, and that $\lambda_{2}(x)<f(0)<\lambda_{1}(x)$. Hence, $\lambda_{2}$ has a strictly negative limit and we deduce that $y_{\tau}^{\prime}(x) \rightarrow 0$. Furthermore, since $\lambda_{1}$ has a finite limit we get that $y_{\tau}^{\prime \prime}$ also has a limit. Then $y_{\tau}^{\prime \prime}(x) \rightarrow 0$. It follows that

$$
\lim _{x \rightarrow+\infty} \lambda_{1}=-\tanh (c), \quad \lim _{x \rightarrow+\infty} \lambda_{2}=-\operatorname{coth}(c) .
$$

We conclude that the cylinder $C_{c}$ is an $f$-surface. Observe that, until now, we have not used the hypothesis $f^{\prime} \geq 0$. As $f$ is increasing, we know that the function

$$
g(t)=\frac{\tanh (t)+\operatorname{coth}(t)}{2}+f\left(\left[\frac{\tanh (t)-\operatorname{coth}(t)}{2}\right]^{2}\right)
$$

is decreasing. Consequently, $t<c$ implies

$$
-f\left(\left[\frac{\tanh (t)-\operatorname{coth}(t)}{2}\right]^{2}\right)<\frac{\tanh (t)+\operatorname{coth}(t)}{2} .
$$

Also let us note that the above observations about the principal curvatures show that $\lambda_{1}-\lambda_{2}$ is a decreasing function for $x>0$. We deduce that $\left[\frac{\lambda_{1}-\lambda_{2}}{2}\right]^{2}$ is also a 
decreasing function. Then for every $x>0$ we have

$$
\left[\frac{\lambda_{1}-\lambda_{2}}{2}\right]^{2}>\left[\frac{\tanh (c)-\operatorname{coth}(c)}{2}\right]^{2},
$$

from which we derive

$$
-f\left(\left[\frac{\lambda_{1}-\lambda_{2}}{2}\right]^{2}\right)<\sqrt{\left[\frac{\lambda_{1}-\lambda_{2}}{2}\right]^{2}+1}
$$

for $x>0$, since

$$
\frac{\tanh (t)+\operatorname{coth}(t)}{2}=\sqrt{\left[\frac{\tanh (t)-\operatorname{coth}(t)}{2}\right]^{2}+1}
$$

The last inequality implies that

$$
0<\frac{\lambda_{1}+\lambda_{2}}{2}+\sqrt{\left[\frac{\lambda_{1}-\lambda_{2}}{2}\right]^{2}+1} .
$$

A computation shows that this is equivalent, for $x$ big enough, to $\lambda_{1} \cdot \lambda_{2}<1$. Replacing $\lambda_{1}$ and $\lambda_{2}$ in the last inequality by their expressions as functions of $y_{\tau}$ and doing further simplifications, we get, for $x$ big enough,

$$
0<y_{\tau}^{\prime \prime} \cosh ^{3}\left(y_{\tau}\right)+y_{\tau}^{\prime 4} \sinh \left(y_{\tau}\right) \text {. }
$$

On the other hand, note that $y_{\tau}^{\prime}(x) \cosh \left(y_{\tau}(x)\right)>0$ for every $x>0$, and moreover

$$
y_{\tau}^{\prime}(0) \cosh \left(y_{\tau}(0)\right)=0 \quad \text { and } \quad \lim _{x \rightarrow+\infty} y_{\tau}^{\prime}(x) \cosh \left(y_{\tau}(x)\right)=0 .
$$

From the above, we deduce that there is a sequence $x_{n}>0$, with $\lim _{n \rightarrow+\infty} x_{n}=$ $+\infty$, such that

$$
\left(y_{\tau}^{\prime} \cosh \left(y_{\tau}\right)\right)^{\prime}\left(x_{n}\right)<0 .
$$

This means that $\left(y_{\tau}^{\prime \prime} \cosh \left(y_{\tau}\right)\right)\left(x_{n}\right)<-\left(y_{\tau}^{\prime 2} \sinh \left(y_{\tau}\right)\right)\left(x_{n}\right)$. Then, for every $n$ :

$$
\left(y_{\tau}^{\prime \prime} \cosh ^{3}\left(y_{\tau}\right)+y_{\tau}^{\prime 4} \sinh \left(y_{\tau}\right)\right)\left(x_{n}\right)<\left(\sinh \left(y_{\tau}\right) y_{\tau}^{\prime 2}\left(y_{\tau}^{\prime 2}-\cosh ^{2}\left(y_{\tau}\right)\right)\left(x_{n}\right)\right.
$$

which is $<0$ for $n$ big enough. But this gives a contradiction with $(*)$. We conclude that $y_{\tau}$ has to be periodic, as desired.

For the remaining cases, $-1 \leq f(0) \leq 0$, we need to consider the angle $\sigma \in$ ] $-\pi / 2, \pi / 2$ [ between $\gamma_{\tau}$ and the coordinate curves $\{y=$ const $\}$. Notice that the orientation chosen is such that $\cos \sigma=\cosh (y) /\left(\cosh ^{2}(y)+y^{\prime 2}\right)^{1 / 2}$. It follows that

$$
\lambda_{2}(x)=\frac{-\cos \sigma}{\tanh (y)} .
$$

Proof of Statement (2). Let us suppose now that $f(0)=-1$ (and $f^{\prime} \geq 0$ ). Therefore $x_{\tau}=+\infty$ or, equivalently, $y_{\tau}$ is defined on $]-\infty,+\infty[$. For otherwise, using the family of horocycles issuing from the point $(u, v)=(1,0)$ in $\partial \mathbf{D}$, we may reach a tangent point between $\gamma_{\tau}$ and one of those horocycles with $\gamma_{\tau}$ on one side of the horocycle. But those two curves generate two $f$-surfaces, so we would have a contradiction with the maximum principle. Observe also that if $\lim _{x \rightarrow+\infty} y_{\tau}(x)=c<+\infty$, the cylinder $C_{c}$ would be an $f$-surface. This gives a contradiction, since the mean curvature of $C_{c}$ is strictly less than -1 (with respect to the exterior normal orientation), and $f \geq-1$ because $f$ is increasing and $f(0)=-1$. So this allows us to conclude that $\lim _{x \rightarrow+\infty} y_{\tau}(x)=+\infty$. As $\lambda_{2}(x)$ is a strictly increasing function and bounded from above by -1 , we deduce that $\lim _{x \rightarrow+\infty} \lambda_{2}=\lim _{x \rightarrow+\infty}-\cos \sigma \leq-1$; hence $\lim _{x \rightarrow+\infty} \cos \sigma=1$. This means that $\gamma_{\tau}$ is tangent to $\partial \mathbf{D}$ when $x$ goes to $\pm \infty$. 
We also have that $\lim _{x \rightarrow+\infty} \lambda_{2}=-1$. Since the function

$$
G\left(\lambda_{1}, \lambda_{2}\right)=\frac{\lambda_{1}+\lambda_{2}}{2}-f\left(\left[\frac{\lambda_{1}-\lambda_{2}}{2}\right]^{2}\right)
$$

is increasing with respect to $\lambda_{1}$ and $\lambda_{2}$ with $G(f(0), f(0))=0$, we also deduce that $\lim _{x \rightarrow+\infty} \lambda_{1}=-1$. We conclude that $M_{\tau}$ is asymptotically umbilic.

Proof of Statement (1). Let us assume $-1<f(0) \leq 0$ (and $f^{\prime} \geq 0$ ). Consider the family of umbilic $f$-surfaces of $\mathbf{H}^{3}$ invariant under rotation about the $y$-axis. Namely, this is the family of pieces of sphere making an angle $\alpha \in] 0, \pi / 2$ ] with $\partial \mathbf{H}^{3}$ such that $\cos (\alpha)=-f(0)$ and each spherical piece stands above the totally geodesic plane with the same boundary. Suppose that $x_{\tau}=+\infty$. Assume first that $\lim _{x \rightarrow+\infty} y_{\tau}(x)=+\infty$. Then we could find one of the previous spherical pieces tangent to and above $M_{\tau}$, which contradicts the maximum principle. If $\lim _{x \rightarrow+\infty} y_{\tau}(x)=c$ with $c<+\infty$, we would conclude as before that the cylinder $C_{c}$ is an $f$-surface. But any cylinder has mean curvature strictly less than -1 (with respect to the outward orientation), and $f$ is bigger than -1 . So we deduce that $x_{\tau}<+\infty$. As we know that $\gamma_{\tau}$ is a complete curve, we have $\lim _{x \rightarrow x_{\tau}} y_{\tau}=+\infty$. As before, since $\lambda_{2}$ has a finite limit at $x_{\tau}$, we conclude that $\cos \sigma$ also has a limit at $x_{\tau}$. Let us call $\sigma_{\tau}$ the limit angle. This means that $\gamma_{\tau}$ has a limit tangent direction and that the angle between $\gamma_{\tau}$ and $\partial \mathbf{D}$ is $\sigma_{\tau}$.

Using the family of umbilic $f$-surfaces (with respect to the outward normal orientation) invariant under rotation about the $x$-axis, the maximum principle shows that $\sigma_{\tau} \leq \alpha$. Moreover, using the first family of umbilic $f$-surfaces (namely, those invariant under rotation about the $y$-axis), the maximum principle shows that $\sigma_{\tau} \geq \alpha$. We obtain that $\sigma_{\tau}=\alpha$; hence $\cos \left(\sigma_{\tau}\right)=-f(0)$. As $\lambda_{2}$ goes to $-\cos \left(\sigma_{\tau}\right)$ when $x$ goes to $x_{\tau}$, we deduce that $\lim _{x \rightarrow x_{\tau}} \lambda_{2}=f(0)$. The same argument as in the case $f(0)=-1$ shows that we also have $\lim _{x \rightarrow x_{\tau}} \lambda_{1}=f(0)$. Thus, $M_{\tau}$ is again asymptotically umbilic.

Finally, assume that $-1 \leq f \leq 0$. Observe that in this case there is no $f$ special cylinder, since the mean curvature of any cylinder is strictly less than -1 . Therefore, we can follow the same proof as before for the cases $f(0)=-1$ and $-1<f(0) \leq 0$. This concludes the proof of Theorem 2 .

Remark 6. (a) It is easy to show that if a plane curve which attains a minimum $(0, \tau), \tau>0$, generates an $f$-surface of revolution $M$, then $\tau$ satisfies the conditions of Theorem 2 (so our hypothesis are necessary). Therefore, the proof of the first part of Theorem 2 also shows that $M$ can be extended as a complete embedded $f$-surface of revolution. Namely, $M$ is part of one surface $M_{\tau}$ given by Theorem 2 .

(b) In the case $f(0)<-1$, for the geometrical description of $\gamma_{\tau}$, note that we only used the fact that $g$ is a decreasing function. It follows that we only need the hypothesis $f^{\prime}(t)>-1 / 2 \sqrt{t+1}$ to show that $\gamma_{\tau}$ is periodic.

In order to prove uniqueness of $f$-surfaces given by Theorem 2 , we shall need the following lemma.

Lemma 7. Let $f$ be an elliptic function with $f^{\prime} \geq 0$. Let $y=y(x)$ be a $C^{2}$ decreasing and strictly positive function defined on an interval $] x_{0},+\infty[$. Then the surface of revolution $M$ generated by the graph of $y(x)$ is not an $f$-surface. 
Proof. Let $c=\lim _{x \rightarrow+\infty} y(x)$. We have $0 \leq c<+\infty$. Let us suppose that $M$ is an $f$-surface. Observe that Lemma 6 implies that the principal curvatures of $M$ are monotone functions, and that $\lambda_{1}$ is increasing if and only if $\lambda_{2}$ is decreasing.

Let us first suppose $c=0$. Therefore $y^{\prime}$ does not have $-\infty$ as limit when $x$ goes to $+\infty$. We deduce that $\lim _{x \rightarrow+\infty} \lambda_{2}=-\infty$. It follows that $\lambda_{2}$ is decreasing, so $\lim _{x \rightarrow+\infty}\left(\lambda_{2}-\lambda_{1}\right)=-\infty$. Note that

$$
-\lambda_{1}=\frac{\lambda_{2}-\lambda_{1}}{2}-f\left(\left[\frac{\lambda_{2}-\lambda_{1}}{2}\right]^{2}\right) .
$$

Then

$$
\lim _{x \rightarrow+\infty} \lambda_{1}=\lim _{t \rightarrow+\infty}\left(t+f\left(t^{2}\right)\right) .
$$

As $f$ is increasing, it follows that $\lim _{x \rightarrow+\infty} \lambda_{1}(x)=+\infty$. Consequently the expression of $\lambda_{1}$ in terms of $y$ implies that $\lim _{x \rightarrow+\infty} y^{\prime \prime}(x)=+\infty$. But the last fact yields a contradiction, because $y^{\prime}$ is negative. Hence, $c \neq 0$.

Let us assume now that $c>0$. As $\lim _{x \rightarrow+\infty} y^{\prime} \neq-\infty$ and $\lambda_{2}$ has a limit, we conclude that $y^{\prime}$ has a finite limit, and then $\lim _{x \rightarrow+\infty} y^{\prime}=0$. Similarly, we can show that $\lim _{x \rightarrow+\infty} y^{\prime \prime}(x)=0$. It follows that

$$
\lim _{x \rightarrow+\infty} \lambda_{1}(x)=-\tanh (c), \quad \lim _{x \rightarrow+\infty} \lambda_{2}(x)=-\operatorname{coth}(c) .
$$

Consequently, we have $\lambda_{1}(x)>\lambda_{2}(x)$, and Lemma 6 shows that $\lambda_{2}$ is decreasing and $\lambda_{1}(x)$ is increasing. Hence $\left[\lambda_{1}(x)-\lambda_{2}(x)\right]^{2}$ is an increasing function, and we get $\left[\lambda_{1}(x)-\lambda_{2}(x)\right]^{2}<[\tanh (c)-\operatorname{coth}(c)]^{2}$. As $f$ is increasing, we have

$$
f\left(\left[\frac{\lambda_{1}(x)-\lambda_{2}(x)}{2}\right]^{2}\right)<f\left(\left[\frac{\tanh (c)-\operatorname{coth}(c)}{2}\right]^{2}\right) .
$$

On the other hand, a continuity argument shows that the cylinder $C_{c}$ is an $f$-surface. This allows us to conclude that

$$
\frac{\lambda_{1}(x)+\lambda_{2}(x)}{2}<\frac{-\tanh (c)-\operatorname{coth}(c)}{2} .
$$

Hence the mean curvature of $M$ is smaller than the mean curvature of $C_{c}$, with respect to the outward normal orientation. But it is well-known (see [8] and [11]) that for every $H<-1$ there exists a family of complete embedded Delaunay type surfaces of revolution with constant mean curvature $H$. This means that those surfaces are periodic and vary continuously. Now, it is also well-known (see [12], Lemma 6.4, or [15], Corollary 4.1.1) that by doing a little perturbation of $C_{c}$ in this family, we can get a surface with the same constant mean curvature as $C_{c}$ which is tangent to and stays under $M$. As the mean curvature of $M$ is smaller, this gives a contradiction with the usual maximum principle (that is, the maximum principle for mean curvature). Thus $M$ cannot be an $f$-surface.

Remark 7. Observe that Lemma 7 remains true if we replace the hypothesis $f^{\prime} \geq 0$ by $|f| \leq 1$. Indeed, we would again have $\lim _{t \rightarrow+\infty}\left(t+f\left(t^{2}\right)\right)=+\infty$, so this eliminates the case $c=0$ (keeping the notation of the proof). Observe also that, in this case, the mean curvature of any $f$-surface is always bigger than -1 and smaller than 1 . Then there is no $f$-special cylinder. This eliminates the case $c>0$.

Let $M$ be a complete embedded non-compact surface of revolution in $\mathbf{H}^{3}$ which does not intersects the axis of rotation ( $x$-axis). Hence, $\mathbf{H}^{3}-M$ has two connected components, one of which contains the $x$-axis. Then we call the unit normal field 
along $M$ pointing toward the component which does not contain the $x$-axis the exterior normal orientation. Observe that this definition coincides with the outward normal orientation in the case when $M$ is generated by a graph.

Theorem 3. Let $f$ be an elliptic function satisfying one of the two following conditions:

(1) $f^{\prime} \geq 0$ and $f(0) \leq 0$;

(2) $-1 \leq f \leq 0$.

Let $M$ be a complete and embedded $f$-surface of revolution that is not totally umbilic.

Then $M$ is one of the surfaces given by Theorem 2.

Proof. Let us assume first that $f$ satisfies condition (1).

Let $\gamma \subset \mathbf{D}$ be the curve generating $M$. Observe that if $\gamma$ intersects the $x$-axis then, for regularity, this intersection must be orthogonal. But this point would be an umbilic point. Hence, as $M$ is not a totally umbilic surface, Lemma 5 shows that this situation is impossible. So we can suppose that $\gamma$ stays in the part $\{y>0\}$ of D.

Let us prove that $\gamma$ is a graph. If it were not, $\gamma$ would have a vertical point $p$ (this means the tangent of $\gamma$ at $p$ is vertical). Let $\gamma^{-}$be the component of $\gamma-p$ which begins under $p$. Up to a symmetry, we can suppose that a neighborhood of $\gamma^{-}$near $p$ stays in the region $\{x \geq x(p)\}$. Observe that the $y$-coordinate of $\gamma^{-}$ is decreasing near $p$. Suppose that $\gamma^{-}$has a horizontal point (this means a point where the tangent is horizontal), and let $q$ be the first one. Lemma 4 implies that $\gamma$ is symmetric with respect to the vertical geodesic $\{x=x(q)\}$, and then $q$ is a local minimum. Observe that, if $\gamma^{-}$had another horizontal point, then the next such point after $q$ would be a local maximum. Hence $\gamma$ would be a periodic curve (see Lemma 4) and $q$ a global minimum for $\gamma$. This implies that the exterior normal orientation of $\gamma$ (or $M$ ) at $q$ points in the direction of increasing $y$. But then near $q$ the curve $\gamma$ is a graph with $q$ as minimum. As $\gamma$ generates an $f$-surface $M$, the proof of Theorem 2 shows that $M$ should be one of the surfaces given there (see Remark 6(a)). But this is absurd, since no surface given by Theorem 2 is generated by a curve which admits a vertical point. So $\gamma^{-}$has no other horizontal point after $q$. Hence we deduce that $q$ is a global minimum for $\gamma$, which is absurd as we have seen before. It follows that $\gamma^{-}$cannot have a horizontal point after $p$.

Now if $\gamma^{-}$were a graph, this graph would be decreasing and defined on the interval $] x(p),+\infty[$ (as $\gamma$ is complete). But Lemma 7 shows this is not possible. So we conclude that $\gamma^{-}$is not a graph. This allows us to deduce that $\gamma^{-}$has another vertical point after $p$. Let $p^{\prime}$ be the first one. Note that hence $\gamma^{-}$is a decreasing graph between $p$ and $p^{\prime}$. Combining this with Lemma 6 , we find that $\lambda_{2}$ is strictly monotone between those two vertical points. Now, as $y(p), y\left(p^{\prime}\right)>0$, we have $\lambda_{2}(p)=\lambda_{2}\left(p^{\prime}\right)=0$, which gives a contradiction.

Thus $\gamma$ is a complete graph. If $\gamma$ had no horizontal points, then, up to a symmetry, we could assume that $\gamma$ is a decreasing graph defined on an interval ]$x_{0},+\infty\left[,-\infty \leq x_{0}\right.$. But Lemma 7 shows this is impossible. If $\gamma$ had a unique horizontal point, $\gamma$ would be symmetric (see Lemma 4), and then this point would be a global maximum or minimum. Lemma 7 shows that this point cannot be a global maximum. Therefore $\gamma$ admits a global minimum, and hence $M$ is one of the surfaces given by Theorem 2 (see Remark 6(a)). Finally, if $\gamma$ has many horizontal 
points, Lemma 4 shows that $\gamma$ is periodic and so admits a global minimum, which concludes the proof in the first case.

In the second case the proof is analogous, since the principal tool, Lemma 7, is again true in this new context. See Remark 7.

Remark 8. In the euclidean case we do not need to assume $f(0) \leq 0$. This is implied by the other hypothesis in Theorem 3; see [16]. In the hyperbolic case we must assume this hypothesis, since J. Gomes [8] has shown existence of an embedded complete surface of revolution in $\mathbf{H}^{3}$ with constant and strictly positive mean curvature (with respect to the exterior normal orientation).

\section{REFERENCES}

1. J. L. Barbosa, R. Sa Earp. New results on prescribed mean curvature hypersurfaces in space forms. An Acad. Bras. Ci., 67, 1,1-5, (1995).

2. J. L. Barbosa, R. Sa Earp. Prescribed mean curvature hypersurfaces in $H^{n+1}(-1)$ with convex planar boundary I. Geom. Dedicata, 71, 61-74, (1998). MR 99d:53064

3. F.G. Braga Brito, R. Sa Earp. On the structure of certain Weingarten surfaces with boundary a circle. An. Fac. Sci. Toulouse, VI, No 2, 243-255, (1997). MR 99a:53005

4. R. Bryant. Complex analysis and a class of Weingarten surfaces. Preprint.

5. S.S. Chern. On special $W$-surface. Proc. Amer. Math. Soc., 6, 783-786, (1955). MR 17:657h

6. M.P. Do Carmo, H.B. Lawson Jr. On Alexandrov-Bernstein theorems in hyperbolic space. Duke Math. J., 50, 995-1003, (1983). MR 85f:53009

7. M.P. Do Carmo, J.M. Gomes, G. Thorbergsson. The influence of the boundary behaviour on hypersurfaces with constant mean curvature in $H^{n+1}$. Comm. Math. Helvitici, 61, 429-491, (1986). MR 87j:53087

8. J.M. Gomes. Sobre hirpersuperfícies com curvatura média constante no espaço hiperbólico. Tese de doutorado. IMPA, (1985).

9. P. Hartman, W. Wintner. Umbilical points and $W$-surfaces. Amer. J. Math., 76, 502-508, (1954). MR 16:68a

10. H. Hopf. Differential geometry in the large. Lecture Notes in Math., Springer-Verlag 1000, (1983). MR 85b:53001

11. W.Y. Hsiang. On generalization of theorems of A. D. Alexandrov and C. Delaunay on hypersurfaces of constant mean curvature. Duke Math. J., 49, 485-496, (1982). MR 84k:53007

12. N.J. Korevaar, R. Kusner, W.H. Meeks III, B. Solomon. Constant mean curvature surfaces in hyperbolic space. Amer. J. Math., 114, 1-43, (1992). MR 92k:53116

13. G. Levitt, H. Rosenberg. Symmetry of constant mean curvature hypersurfaces in hyperbolic space. Duke Math. J., 52, 53-59, (1985). MR 86h:53063

14. B. Nelli, H. Rosenberg. Some remarks on embedded hypersurfaces in Hyperbolic Space of constant mean curvature and spherical boundary. Ann. Global Anal. Geom., 13, 23-30, (1995). MR 96c:53097

15. H. Rosenberg, R. Sa Earp. The geometry of properly embedded special surfaces in $\mathbf{R}^{3}$, e.g., surfaces satisfying $a H+b K=1$, where $a$ and $b$ are positive. Duke Math. J., 73, 291-306, (1994). MR 95b:53010

16. R. Sa Earp et E. Toubiana. A note on special surfaces in $\mathbf{R}^{3}$. Mat. Contemp., 4, 108-118, (1993). MR 95j:53008

17. R. Sa Earp et E. Toubiana. Sur les surfaces de Weingarten spéciales de type minimal. Bol. Soc. Bras. Mat., 26, No. 2, 129-148, (1995). MR 96j:53006

18. R. Sa Earp et E. Toubiana. Classification des surfaces de type Delaunay et applications. Amer. J. Math. 221, 671-700, (1999).

Departamento de Matemática, Pontifícia Universidade Católica, Rua Marquês de São Vicente, 225, 22453-900 Gávea, Rio de Janeiro, Brasil

E-mail address: earp@mat.puc-rio.br

Département de Mathématiques, Université Paris ViI, 2, Place Jussieu, 75251 Paris Cedex 05, France

E-mail address: toubiana@math.jussieu.fr 